\title{
5D Data Storage by Ultrafast Laser Nanostructuring in Glass
}

\author{
Jingyu Zhang*, Mindaugas Gecevičius, Martynas Beresna, Peter G. Kazansky \\ Optoelectronics Research Centre, University of Southampton, SO17 1BJ, United Kingdom \\ jz2e11@orc.soton.ac.uk
}

\begin{abstract}
The high-density five dimensional data storage with ultrafast laser writing is demonstrated. The text file is recorded by polarization controlled self-assembled form birefringence and retrieved in glass opening the era of unlimited lifetime data storage.

(C) 2013 Optical Society of America

OCIS codes (140.3390) Laser materials processing, (210.0210) Optical data storage
\end{abstract}

The idea of the optical memory based on femtosecond laser writing in the bulk of transparent material was first proposed in 1996 [1,2]. More recently self-assembled nanogratings produced by ultrafast laser writing in glass were proposed for the polarization multiplexed optical memory, where the information encoding would be realized by means of two birefringence parameters, i.e. the slow axis orientation (4th dimension) and strength of retardance (5th dimension), in addition to three spatial coordinates [3,4]. The slow axis orientation and the retardance can be controlled by polarization and intensity of the incident beam respectively [5]. The unprecedented parameters including $360 \mathrm{~TB} /$ disc data capacity, thermal stability up to $1000^{\circ} \mathrm{C}$ and practically unlimited lifetime [6,7]. However the implementation of digital data storage, which is a crucial step towards the real world applications, has not been demonstrated by ultrafast laser writing. Here we successfully recorded and retrieved a digital copy of the text file in 5D using polarization controlled selfassembled ultrafast laser nanostructuring in silica glass.

The experiments were performed with a femtosecond laser system Pharos (Light Conversion Ltd.) operating at $1030 \mathrm{~nm}$ and delivering $8 \mu \mathrm{J}$ pulses of $280 \mathrm{fs}$ at $200 \mathrm{kHz}$ repetition rate. The intensity distribution at the focal plane was modulated via a spatial light modulator (SLM), which split the incident light into 256 beams. The hologram generated on the SLM was reimaged via a 4-f optical system on the back pupil of the objective (Fig. 1 (a)). In addition, a half-wave plate matrix, imprinted by the laser nanostructuring of fused silica, was added to the 4-f optical system for the motion free polarization control. The laser beam was focused with a $1.2 \mathrm{NA}$ water immersion microscope objective at the depth of $140 \mu \mathrm{m}$ below the surface of the silica glass sample mounted on a three-axial translation stage (ABL1000, Aerotech Ltd.).

(a)

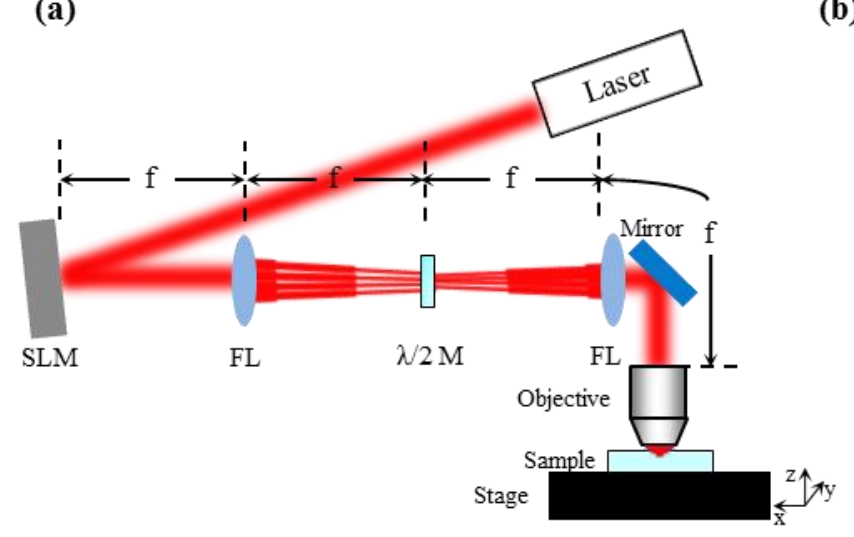

(b)

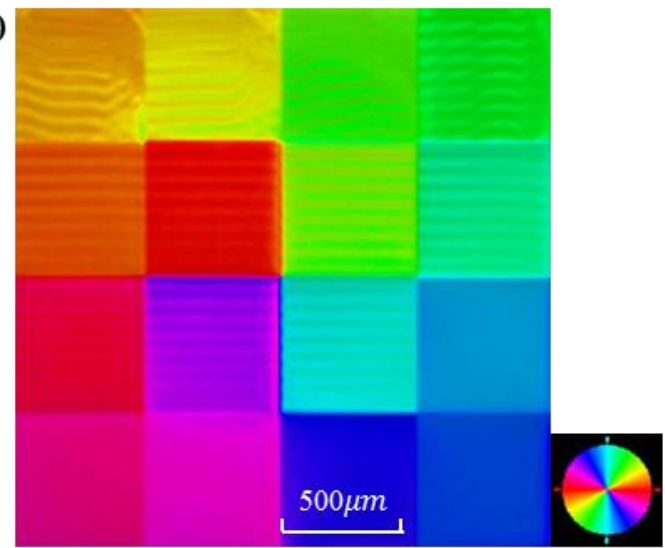

Fig. 1. (Color online) (a) 5D optical storage writing setup: femtosecond laser, spatial light modulator (SLM), Fourier lens (FL), half-wave plates matrix $(\lambda / 2 \mathrm{M})$, dichroic mirror, 1.2 NA water immersion objective, silica glass sample, translation stage. (b) Color-coded slow axis orientation of the half wave-plates matrix imprinted in silica glass.

The combination of the SLM and a half-wave plate matrix allowed the removal of relatively slow rotating and moving components for retardance and slow axis orientation control (Fig. 1 (a)). An adapted weighted Gerchberg-Saxton algorithm was used to set the split beam energy at several levels at the back focal plane of the objective [3]. Combined with a phase distribution of Fresnel lens, various levels of intensity at different depths of the focal plane could be achieved. The polarization direction was controlled by the half-wave plates 
matrix (Fig. 1 (b)), where beams passing through the selected segment can generate the targeted polarization state.

For the demonstration of this technology, the copy of this abstract was recorded into two different levels of retardance ( 1 bit) and four slow axis orientations ( 2 bits). As a result each laser imprinted spot stores 3 bits of information. The digital copy of the recoded text was divided into the matrix in three planes written at different depth with the distance of $5 \mu \mathrm{m}$ between the planes (Fig. 2 (a)).

The read-out of the recorded text file was achieved by a BX51 (Olympus Inc.) optical microscope based quantitative birefringence measurement system (CRi Abrio). Both the slow axis and retardance distributions of the birefringent spot array were successfully retrieved after each sequence (Fig. 2 (b)).

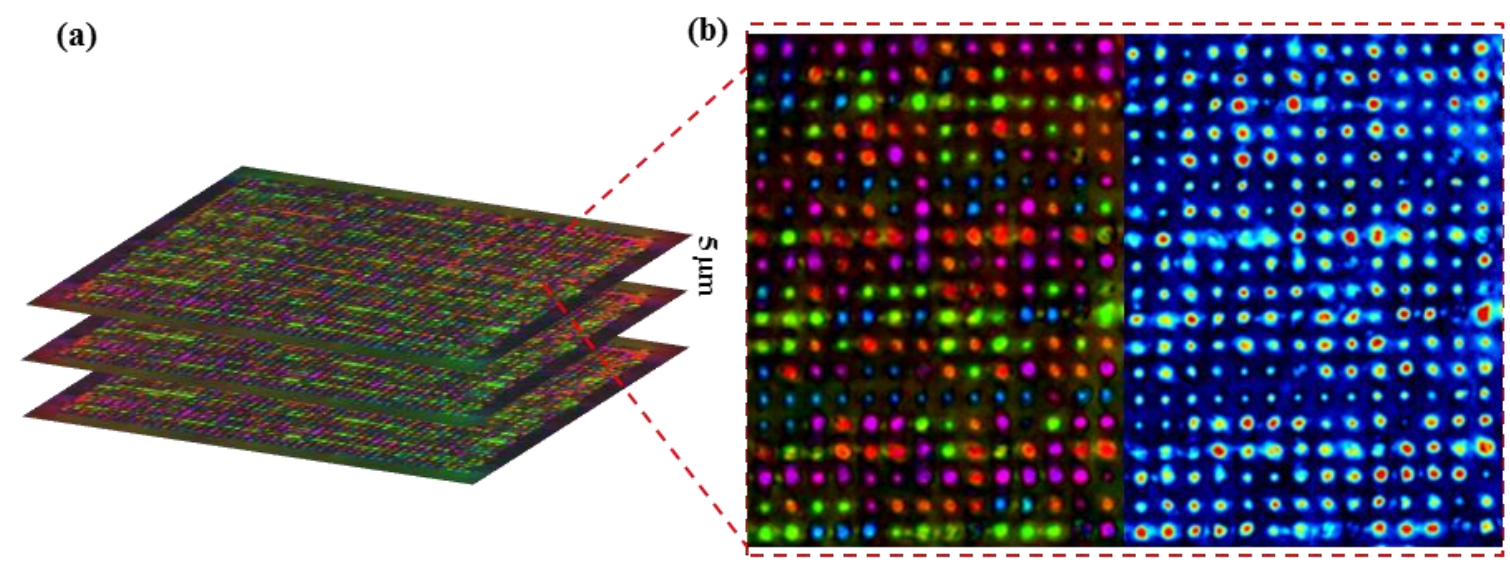

Fig. 2. (Color online) (a) Birefringence measurement of the data recorded in three separate layers. (b) slow axis (left) and retardance (right) distribution measured with $60 \times$ magnification.

In conclusion, we experimentally demonstrated the recording and read-out processes of 5D optical data by femtosecond laser writing. The data recording was significantly simplified by replacing the conventional control of the writing beam energy and polarization with a spatial light modulator and a specially designed laser imprinted half-wave plate matrix. This demonstration is a crucial step towards commercialization of ultrafast laser based optical data storage.

\section{References}

1. E. N. Glezer, M. Milosavljevic, L. Huang, R. J. Finlay, T. H. Her, J. P. Callan, and E. Mazur, "Threedimensional optical storage inside transparent materials.," Optics Letters 21, 2023-2025 (1996).

2. P. Zijlstra, J. W. M. Chon, and M. Gu, "Five-dimensional optical recording mediated by surface plasmons in gold nanorods.," Nature 459, 410 (2009).

3. Y. Shimotsuma, M. Sakakura, P. G. Kazansky, M. Beresna, J. Qiu, J. Qiu, K. Miura, and K. Hirao, "Ultrafast manipulation of self-assembled form birefringence in glass," Advanced Materials 22, 4039-4043 (2010).

4. M. Beresna, M. Gecevičius, P. G. Kazansky, T. Taylor, and A. V. Kavokin, "Exciton mediated self-organization in glass driven by ultrashort light pulses," Applied Physics Letters 101, 053120 (2012).

5. E. Bricchi, B. G. Klappauf, and P. G. Kazansky, "Form birefringence and negative index change created by femtosecond direct writing in transparent materials," Optics Letters 29, 119-121 (2004).

6. E. Bricchi and P. G. Kazansky, "Extraordinary stability of anisotropic femtosecond direct-written structures embedded in silica glass," Applied Physics Letters 88, 111119 (2006).

7. R. S. Taylor, C. Hnatovsky, E. Simova, P. P. Rajeev, D. M. Rayner, and P. B. Corkum, "Femtosecond laser erasing and rewriting of self-organized planar nanocracks in fused silica glass," Optics Letters 32, 2888 (2007). 